\title{
DETECTION AND STATION MAPPING OF MEDIASTINAL LYMPH NODES ON THORACIC COMPUTED TOMOGRAPHY USING SPATIAL PRIOR FROM MULTI-ATLAS LABEL FUSION
}

\author{
Jiamin Liu, Jocelyn Zhao, Joanne Hoffman, Jianhua Yao, Le Lu, Evrim B. Turkbey, \\ Christine Kim, Ronald M. Summers \\ Imaging Biomarkers and Computer-aided Diagnosis Laboratory \\ Radiology and Imaging Sciences, National Institutes of Health Clinical Center \\ Building 10 Room 1C224 MSC 1182, Bethesda, MD 20892-1182
}

\begin{abstract}
Lymph nodes play an important role in clinical practice but detection is challenging due to low contrast surrounding structures and variable size and shape. In this paper, we propose a fully automatic method for mediastinal lymph node detection and station mapping on thoracic CT scans. First, lungs are automatically segmented to locate the mediastinum region. Shape features by Hessian analysis, local scale, and circular transformation are computed at each voxel. Spatial prior distribution is determined based on the identification of 11 anatomical structures by using multiatlas label fusion. Shape features and spatial prior are then integrated for lymph node detection. The detected candidates are segmented by curve evolution. Characteristic features are calculated on the segmented lymph nodes and support vector machine is utilized for classification and false positive reduction. We applied our method to 20 patients with 62 enlarged mediastinal lymph nodes. The system achieved a significant improvement with $80 \%$ sensitivity at 8 false positives per patient with spatial prior compared to $45 \%$ sensitivity at 8 false positives per patient without a spatial prior. With the segmentation of spatial anatomic structures, $88 \%$ of mediastinal lymph nodes are correctly mapped to their stations.
\end{abstract}

Index Terms - lymph node detection, Hessian analysis, spatial prior, multi-atlas label fusion

\section{INTRODUCTION}

In clinical practice, lymph nodes play an important role, especially in the mediastinal area. Many diseases, such as lung cancer, lymphoma and inflammation will cause affected lymph nodes to be enlarged. According to the RECIST guidelines [1], a lymph node is considered enlarged (swollen) if it is more than $10 \mathrm{~mm}$ in diameter (short axis) on computed tomography (CT) images.
Lymph node analysis is a significant part of daily clinical work in radiology. Mediastinal lymph nodes in patients with lung cancer are routinely assessed by clinicians for accurate staging, to determine prognosis and choice of proper therapy, and to identify interval changes on follow-up examinations. This assessment is usually done using thoracic CT. In the current clinical workflow, radiologists and physicians have to detect, quantitatively evaluate and classify lymph nodes by manually examining all slices of CT scans. This process can be very time consuming and errorprone and annotations from different human observers can vary significantly. Therefore, a system for automated lymph node detection and measurement is desirable. However, automatic detection of mediastinal lymph nodes is challenging because lymph nodes have CT attenuation similar to other mediastinal soft tissues such as the esophagus and cardiac musculature and therefore low contrast to surrounding structures. Moreover, their shape and size varies considerably. Examples of mediastinal lymph nodes are shown in Figure 1.

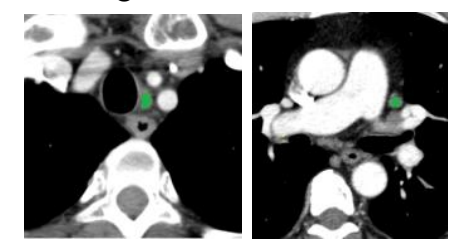

Fig. 1. Two examples of mediastinal lymph nodes (green).

Few works has been published on the automated detection of lymph nodes. Kitasaka et al. [2] proposed a 3D minimum directional difference (Min-DD) filter for automated detection of lymph nodes in abdominal CT images. Hessian-based linear structures (vessels) detection and some morphological operations are used to reduce the false positives. Barbu et al. [3] proposed a machine learning based method for detection and segmentation of axillary and pelvic lymph nodes on CT images. Feuerstein [4] proposed an atlas of lymph node stations and Hessian analysis for automated detection of lymph nodes on chest CT images. 
Feulner [5] used discriminative learning in combination with segmentation of heart and esophagus for lymph node detection on chest CT images.

In this paper, we use shape features in combination with spatial priors from 11 anatomical structures to detect lymph nodes on CT scans. The major contribution of this work is to combine spatial prior anatomical knowledge from segmentation of multi-organ using multi-atlas with local adaptive label fusion. Since single-atlas based segmentation is generally inaccurate because of variations in the anatomy, we use multi-atlas with local adaptive label fusion for segmentation of anatomical structures in the chest.

\section{METHODS}

\subsection{Region of interest}

This work focuses on detecting mediastinal lymph nodes. Therefore, a region of interest (ROI) is automatically detected that covers the mediastinum. This ROI is determined as the convex hull of segmented lungs.

\subsection{Shape features for detection}

\subsubsection{Blobness by local object scale and Hessian analysis}

The object scale $k$ at every voxel is defined as the radius of the largest hyperball centered at the voxel such that all voxels within the ball satisfied a predefined image intensity homogeneity criterion [6]. Thus, object scale represents the size of local structure. Object scale at the center of a bloblike structure approximately equals the radius of the blob. A blobness $B_{\sigma}(x)$ is then defined as:

$$
B_{\sigma}(x)=\left\{\begin{array}{cc}
\left(1-e^{\frac{-R_{A}^{2}}{2}}\right)\left(1-e^{\frac{-S^{2}}{2}}\right) e^{\frac{-R_{c}^{2}}{2}} & \text { if } \lambda_{i}<0 \text { for } i=1,2,3 \\
0 & \text { otherwise }
\end{array}\right.
$$

with $R_{A}=\frac{\left|\lambda_{2}\right|}{\left|\lambda_{3}\right|}, S=\sqrt{\lambda_{1}^{2}+\lambda_{2}^{2}+\lambda_{3}^{2}}$ and $R_{c}=k-2 \sigma \cdot \lambda_{1}, \lambda_{2}, \lambda_{3}$, $\left(\left|\lambda_{1}\right| \leq\left|\lambda_{2}\right| \leq\left|\lambda_{3}\right|\right)$ are the corresponding eigenvalues of Hessian matrix at the voxel. Blobness $B_{\sigma}(x)$ is defined between 0 and 1 and will be maximum when $\sigma, k$, and the radius of the blob approximately match. Ideally, the center of the blob has the maximal value. In our application, blobness is evaluated at $\sigma=2.5,3.5$ and $4.5 \mathrm{~mm}$, and finally the maximal $B_{\sigma}(x)$ is selected for every voxel.

\subsubsection{Circular transformation}

The transformation is termed "circular transformation" because it performs best when a perfect circular or spherical region is present. The circular transformation is designed based on the observation that for a point within a (roughly) circular/spherical-shaped image region, the variation of the distances from the point to the region boundary reaches the minimum when the point lies exactly at the region centroid [7]. For each voxel, a number $(m)$ of evenly-oriented radial line segments of specific length are generated. The circular transform at the voxel $x$ is defined as

$$
C t(x)=\frac{\sum_{i=1}^{n} g_{i}}{\frac{1}{\bar{D}} \sqrt{\sum_{i=1}^{n}\left(D_{i}-\bar{D}\right)^{2}}},
$$

where $g_{i}$ is the local maximal gradient on the $i$ th radial line, $D_{i}$ is the distance from $x$ to the voxel with $g_{i}, \bar{D}$ is the mean of all $D_{i}$. It is apparent that $C t$ will be maximal at the centroid of a circular/spherical object. In this work, radial lines are generated every $20^{\circ}$ on three orthogonal views $(m=54)$.

\subsection{Spatial prior from anatomical organs}

Identification of anatomical organs plays an important role in lymph node analysis. Lymph nodes are not located inside of any organ and this prior knowledge is helpful for detection. In addition, lymph node stations can be determined automatically with respect to anatomical structures.

\subsubsection{Robust structures segmentation}

In this work, we first identify those structures which can be detected robustly in CT scans in the chest area such as trachea, lungs and spine ("group 1" organs). The lungs and the trachea are detected using thresholding followed by morphological operations. The spine is segmented using a watershed algorithm followed by a directed graph search described in [8]. All segmentation methods do not require user interaction.

\subsubsection{Multi-atlas segmentation with label fusion}

Besides the robust "group 1" structures, eight other organs, esophagus, aortic arch, pulmonary trunk, descending aorta, ascending aorta, superior vena cava, azygos arch, and heart ("group 2" organs) are detected by multi-atlas label fusion [9].

Let $U=\left(I_{T}, G_{T}^{1}, G_{T}^{2}\right)$ be a target image to be segmented and $A_{1}=\left(I_{1}, G_{1}^{1}, G_{1}^{2}\right), \ldots, A_{n}=\left(I_{n}, G_{n}^{1}, G_{n}^{2}\right)$ be $n$ atlases. $I_{i}, G_{i}^{1}$ and $G_{i}^{2}$ denote the $i$ th atlas image and its corresponding labels. $G_{i}^{1}$ are the automatically detected "group 1" organs and $G_{i}^{2}$ are the manually labeled "group 2 " organs.

In order to be able to propagate the labels from an atlas to a target image, a registration is needed between them. In this work, each atlas is registered to the target image by using label-guided diffeomorphic registration developed in Advanced Normalization Tools (ANTS) [10], where "group 1 " organs are used for label-guided registration. The registration resulted in $n$ transformations $u_{i}$, describing transformations from $A_{i}$ to $U$. These transformations are used to warp the atlas label $G_{i}^{2}$ to the target $U$. For each 
voxel in the target $U$, each atlas provides an opinion about the label. Finally, the label at voxel $x$ in the target $U$ is determined by

$$
\hat{G}_{T}^{2}(x)=\sum_{i=1}^{n} \omega_{i}(x) u_{i}\left[G_{i}^{2}(x)\right]
$$

$u_{i}\left[G_{i}^{2}(x)\right]$ are the warped $i$ th atlas labels ("group 2" organs) and $\omega_{i}(x)$ is a local weight assigned to the $i$ th atlas with $\sum_{i=1}^{n} \omega_{i}(x)=1$. The local adaptive $\omega_{i}(x)$ is estimated based on the assumption that the atlas image that is more similar to the target image has higher weights [11]:

$$
\omega_{i}(x)=\frac{1}{Z(x)} e^{-\sum_{y \in N(x)}\left(u_{i}\left[\left(I_{i}(y)\right]-I_{T}(y)\right)^{2}\right.}
$$

where $N(x)$ defines a neighborhood around voxel $x$ and $Z(x)$ is a normalization constant.

A binary mask is then created to label regions which cannot contain lymph nodes with 0 and other regions with 1 :

$$
M(x)=\left\{\begin{array}{cc}
0 & \text { if } x \text { is inside any organ } \\
1 & \text { else }
\end{array}\right.
$$

All "group 1" and "group 2" organs are excluded in the mask.

The spatial prior probability of having lymph nodes is defined as the minimum distance of voxel $x$ to the surface of all organs:

$$
S(x)=\min \left(d_{j}(x)\right) \quad 1 \leq j \leq O
$$

where $d_{j}(x)$ is the minimal distance from $x$ to $j$ th organ. $S(x)$ indicates that the gaps between organs have higher probability to have lymph nodes. In this work, we have 5 atlases $(n=5)$ and 11 organs $(O=11)$. Figure 2 shows one atlas and the segmentation of different organs.

\subsection{Shape and spatial prior integration}

Now the binary mask $M$, the blobness $B_{\sigma}$, the circular transformation $C t$, and the spatial prior $S$ are merged into a final detection response of detecting a lymph node at voxel $x$. It is modeled as

$$
D(x)=M(x) \max \left(B_{\sigma}(x)\right) C t(x) S(x)
$$

Voxels with higher detection response are more likely to be lymph nodes. In this work, all voxels with $D(x) \geq 0.7$ are clustered as initial lymph node detections.

\subsection{Segmentation of lymph nodes}

Due to the large number of initial detections (hundreds per patient), a fast segmentation method is preferred. A real time level-set-based curve evolution [12] is implemented in our system. This segmentation algorithm is initially developed for real time video tracking applications. It is also suitable for fast segmentation of large detections from a CAD system.

\subsection{Feature computation and classification}

A comprehensive collection of texture features from the segmented lymph node candidates were computed. The
Haralick Gray-Level Co-occurrence Matrix (GLCM) features are widely used for analyzing image texture. The co-occurrence matrix stores the co-occurrence frequencies of pairs of gray levels at different distances and directions. We calculated the co-occurrence matrices for 4 offset distances and 13 directions on multiple planes, yielding 52 matrices for each lymph node candidate. We then calculated 12 features from the matrix, including energy, entropy, correlation, contrast, variance, sum of mean, inertia, cluster shade, cluster tendency, homogeneity, maximal probability, and inverse variance. Thus, each detected candidate has 624 Haralick GLCM features. We also extracted first order graylevel features including minimum, maximum, mean, standard deviation, skewness and kurtosis. Finally, a support vector machine (SVM) committee [13] was applied for classification.

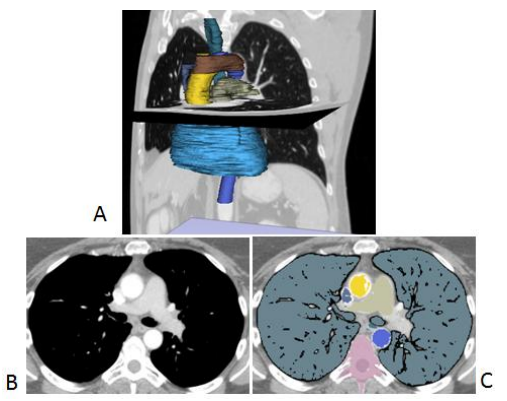

Figure 2. (A) Example of one atlas (3D view with axial \& coronal cut-planes). (B, C) Segmentation of spine (light purple), lungs (light gray), mainstem bronchi (light gray), esophagus (teal), pulmonary trunk (light green), descending aorta (dark purple), ascending aorta (yellow) and superior vena cava (dark gray).

\section{RESULTS}

Our patient population consisted of 25 patients, each having at least one enlarged mediastinal lymph node $(\geq 10 \mathrm{~mm}$ in short axis diameter) on thoracic CT images. The slice thickness was $1 \mathrm{~mm}$, and the voxel spacing within an axial slice was in the range $0.7-0.9 \mathrm{~mm}$. Five patients were used as atlases. For each atlas image, the esophagus, aortic arch, pulmonary trunk, descending aorta, ascending aorta, superior vena cava, and heart ("group 2" organs) were manually labeled. The remaining 20 patients were target images for lymph node detection. The mediastinal lymph nodes in the target images were manually segmented, and the segmentations were reviewed by an experienced radiologist. The mediastinal lymph node stations were also recorded according to an established guideline [14]. In total, 62 enlarged lymph nodes were annotated as ground truth for evaluation.

The detection performance was evaluated using tenfold cross-validation. The free response receiver operating characteristic (FROC) curves of the detection performance are shown in Figure 3. We get a sensitivity of $45 \%$ with 8 FP per patient on average if we only rely on shape characteristics. If the spatial prior is also used, we get a sensitivity of $80 \%$ with 8 FP per patient on average. The 
detection performance is comparable to state of the art methods for mediastinal lymph node detection such as sensitivity of $53 \%$ with 3 FP per patient [5]. Figure 4 shows examples of true and false positive and false negative detections.

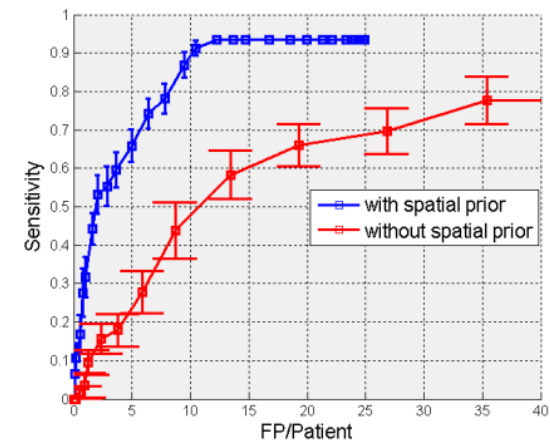

Figure 3. 10-fold cross validation FROC of two methods.

With the segmentation of anatomic structures, lymph node station map in chest area is automated defined according to the guideline [14] and thus the station of detected lymph node is determined automatically. Figure 5 shows an example of lymph node stations based on the segmentation of all 11 organs. Table 1 shows the performance of automated lymph node station mapping. On average, $88 \%$ of mediastinal lymph nodes were correctly mapped to their stations.

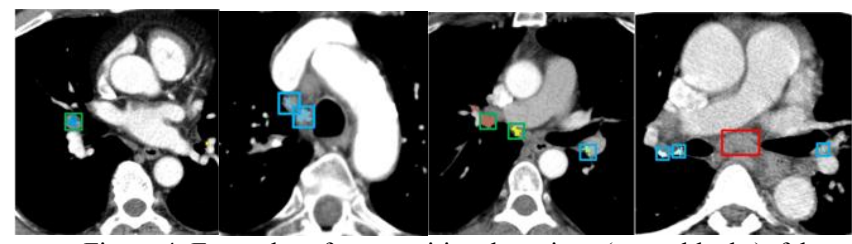

Figure 4. Examples of true positive detections (green blocks), false positive detections (blue blocks) and false negative detections (red block).
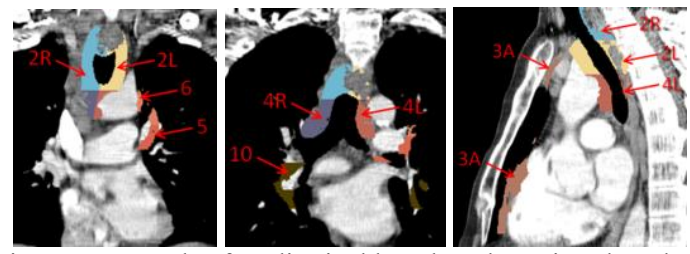

Figure 5. Example of mediastinal lymph node stations based on the segmentation of anatomic structures.

Table 1: Performance of lymph node station mapping. (Stations 7, 8 and 9 are not distinguished.)

\begin{tabular}{|l|l|l|l|l|}
\hline Station & $\begin{array}{l}\text { Number } \\
\text { of nodes }\end{array}$ & Detected & $\begin{array}{l}\text { Correct } \\
\text { Labeled }\end{array}$ & Sensitivity \\
\hline 2L. Upper Paratracheal & 3 & 3 & 3 & $100 \%$ \\
\hline 2R. Upper Paratracheal & 5 & 5 & 5 & $100 \%$ \\
\hline 3A. Pre-vascular & 4 & 2 & 1 & $50 \%$ \\
\hline 3P. Pre-vertebral & No data & - & - & - \\
\hline 4R.Lower Paratracheal & 10 & 8 & 6 & $75 \%$ \\
\hline 4L. Lower Paratracheal & 9 & 9 & 8 & $89 \%$ \\
\hline 5. Subaortic & 4 & 4 & 3 & $75 \%$ \\
\hline 6. Para-aortic & 1 & 1 & 1 & $100 \%$ \\
\hline $\begin{array}{l}\text { 7. Subcarinal } \\
\text { 8. Paraesophageal } \\
\text { 9. Pulmonary Ligament }\end{array}$ & 14 & 14 & 13 & $93 \%$ \\
\hline 10. Hilar nodes & 12 & 12 & 11 & $92 \%$ \\
\hline
\end{tabular}

\section{CONCLUSION}

In this paper, we present a method for automatic detection of mediastinal lymph nodes on thoracic CT images. This is a challenging problem due to the low contrast between lymph nodes and surrounding structures. Shape features and spatial prior from anatomic structures, identified using multi-atlas with local adaptive label fusion, are integrated for lymph node detection and station mapping. Evaluation on 20 patients showed the advantage of the spatial prior. The methods can be adapted for lymph node detection in other anatomic regions, such as the neck, axillae, abdomen and pelvis and also to other node-like lesions such as tumor metastases.

\section{REFERENCE}

1. P. Therasse, etc, "New guidelines to evaluate the response to treatment in solid tumors. European Organization for Research and Treatment of Cancer, National Cancer Institute of the United States, National Cancer Institute of Canada," J Natl Cancer Inst, vol. 92, pp. 205-16, 2000.

2. T. Kitasaka, Y. Tsujimura, Y. Nakamura, K. Mori, Y. Suenaga, M. Ito, and S. Nawano, "Automated extraction of lymph nodes from 3-D abdominal CT images using 3-D minimum directional difference filter," Med Image Comput Comput Assist Interv, vol. 10, pp. 336-43, 2007.

3. A. Barbu, M. Suehling, X. Xu, D. Liu, S. K. Zhou, and D. Comaniciu, "Automatic detection and segmentation of axillary lymph nodes," Med Image Comput Comput Assist Interv, vol. 13, pp. 28-36, 2010.

4. Feuerstein M, Glocker B, Kitasaka T, Nakamura Y, Iwano S, Mori K., "Mediastinal atlas creation from 3-D chest computed tomography images: application to automated detection and station mapping of lymph nodes, " Med Image Anal. 2012 Jan;16(1):63-74.

5, J. Feulner, S. Zhou, M. Huber, J. Hornegger, D. Comaniciu, and A. Cavallaro, "Lymph node detection in 3-D chest CT using a spatial prior probability," CVPR, 2010.

6. Saha, P.K., J.K. Udupa, and D. Odhner, "Scale-based fuzzy connected image segmentation: theory, algorithms, and validation," Computer Vision and Image Understanding, 2000.

7. S. Lu, "Accurate and efficient optic disc detection and segmentation by a circular transformation," IEEE Trans Med Imaging, vol. 30, pp. 212633, 2011.

8. Yao, J, OConnor, SD, Summers, RM, "Automated spinal column extraction and partitioning," Biomedical Imaging: Nano to Macro, IEEE International Symposium on, 390 - 393, 2006.

9. van Rikxoort EM, Isgum I, Arzhaeva Y, Staring M, Klein S, Viergever MA, Pluim JP, van Ginneken B., "Adaptive local multi-atlas segmentation: application to the heart and the caudate nucleus," Med Image Anal. 14(1):39-49, 2010.

10. Avants BB, Epstein CL, Grossman M, Gee JC, Symmetric diffeomorphic image registration with cross-correlation: evaluating automated labeling of elderly and neurodegenerative brain, Med Image Anal. Feb;12(1):26-41,2008.

11. Sabuncu MR, Yeo BT, Van Leemput K, Fischl B, Golland P., "A generative model for image segmentation based on label fusion," IEEE Trans Med Imaging, 29(10):1714-29, 2010.

12. Y. Shi and W. Karl, "A fast level set method without solving PDEs," IEEE International Conference Acoustics Speech and Signal Processing, Philadelphia, PA, 2005.

13. J. Yao, R. M. Summers, and A. K. Hara, "Optimizing the committee of supportvector machines (SVM) in a colonic polyp CAD system," SPIE Medical Imaging, vol. 5746, pp. 384-392, 2005.

14. Rusch VW, Asamura H, Watanabe H, Giroux DJ, Rami-Porta R, Goldstraw P; "The IASLC lung cancer staging project: a proposal for a new international lymph node map in the forthcoming seventh edition of the TNM classification for lung cancer., " J Thorac Oncol. 4(5):568-77, 2009. 